\title{
BIOMATERIALS INHOMOGENEITIES DETECTION BY ELECTROMAGNETIC METHODS
}

The paper deals with the biomaterials nondestructive testing using electromagnetic methods. The eddy current testing and electromagnetic - acoustic transducer methods are analyzed and applied for evaluation of conductive prosthetic materials. The defects detection results achieved by simulations and measurements using both methods are compared and discussed.

\section{Introduction}

Biomaterials are the materials used to manufacture prostheses, implants, and surgical instruments. They have to be completely compatible with the human body and designed not to provoke rejection by our bodies (skin, blood, bone, etc.). The biomaterials which are used in medical praxis are natural (collagen, cellulose called biological biomaterials) or synthetic (metallic, ceramic and glass, polymeric biomaterials). The fails that usually affect the prosthetic replacement result from combination of circumstances including fatigue and stress corrosion. Periodic evaluation of the state of the devices is the significant interest particularly in the case of prosthetics whose failure can be fatal. An example of such a device is the artificial heart valve or total hip replacement. This article presents rather new techniques that have been developed for the detection of defect that occurs in the prosthetic replacements. The main condition for evaluation using electromagnetic methods is that the biomaterials are conductive.

The metallic biomaterials represent the group of materials which could be evaluated using electromagnetic methods. Metals have been used almost exclusively for load-bearing implants, such as hip and knee prostheses (hard tissue replacement). Metals have also been used as parts of artificial heart valves, as vascular stents, and as pacemaker lead (soft tissue replacement). The metallic biomaterials which are currently used in trauma and orthopedic surgery are Ti and its alloys, $\mathrm{CoCr}$ alloys, Stainless steels, dental metals, others.

Special type of soft tissue replacement is an artificial heart valve. Prosthetic heart valves which are used in medical praxis create two main groups. The first are bioprosthetic valves (fabricated from animal or homografts) and the others are the mechanical heart valves. These valves can be constructed using various material combinations such as metal and polymers or plastics, [2].
One special type of mechanical heart valve prostheses is the Bjork-Shiley Convexo Concave (BSCC) heart valve. BSCC heart valve has been used to replace the aortic or mitral valves. Fig. 1 shows typical design of the valve. This type of heart valve is made from metal. Occasionally the weld at one of the ends of the outlet strut develops a fracture and fails due to various reasons including mechanical fatigue and stress corrosion cracking. Although the valve in this condition performs normally, this may be a precursor to a fracture and eventual failure of the other leg of the outer strut, which leads to fatality. Hence, there is a considerable interest in detecting fractures of outlet strut in BSCC heart valves, [1].

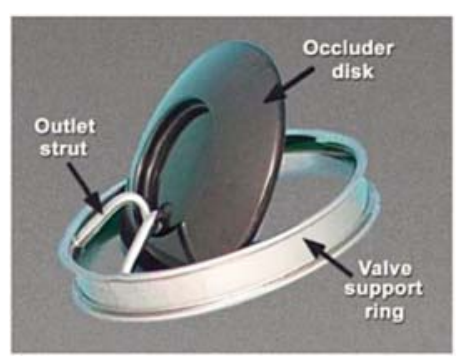

Fig. 1 Typical design of the BSCC heart valve

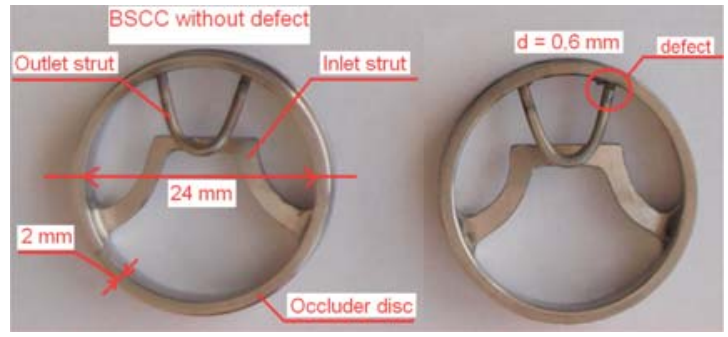

Fig. 2 Prototype of the BSCC heart valve with defect

\footnotetext{
* Tatiana Strapacova, Klara Capova, Milan Smetana

Department of Electromagnetic and Biomedical Engineering, Faculty of Electrical Engineering, University of Zilina, Slovakia,

E- mail: strapacova@fel.uniza.sk
} 
CoCr alloy - Hayness 25 and Stainless steel 316L were used in experimental part. Two possible approaches to establish the biomaterials inhomogeneities are presented in this report. The present paper deals with non-destructive testing using electromagnetic methods for this purpose.

\section{Eddy Current Texting - ECT}

One of the widely utilized electromagnetic methods is the eddy current testing. In this method, the coil is excited by alternating current and eddy currents are induced in conductive object placed in vicinity of the conductive material. It changes the magnetic field around conductive objects where cracks and defects prevent the flow of the eddy currents, and thus leading to changes of the impedance of the pick-up coil. The method is especially suitable for detection of surface and near surface defects. The main advantage of ECT is its high sensitivity to small and shallow defects that occur in prosthetic replacement, [3].

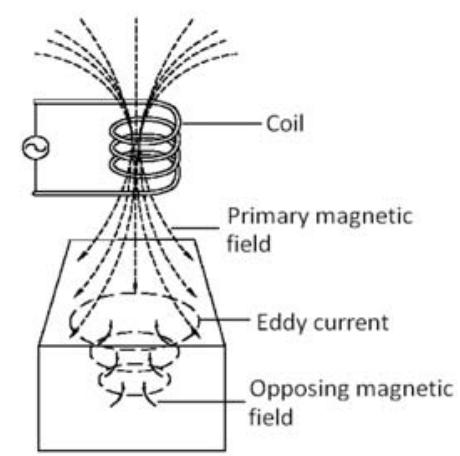

Fig. 3 Fundamental principle of the ECT method

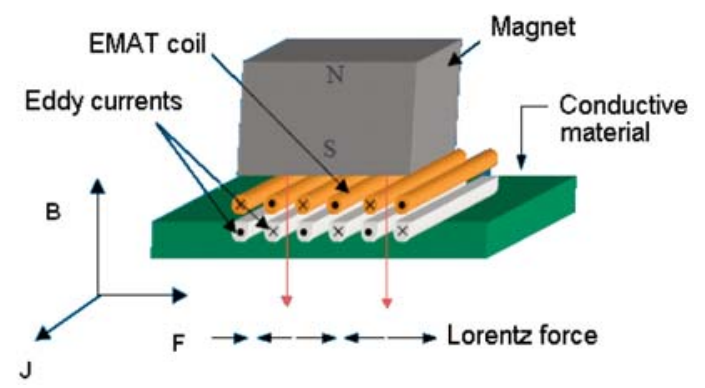

Fig. 4 Ultrasonic waves generation using EMAT

\section{Electromagnetic - Acoustic Transducer Testing - EMAT}

The second approach uses the Electromagnetic - acoustic transducer testing. This transducer converts electromagnetic energy to acoustic energy. When a coil of wire placed near the surface of an electrically conducting object is driven by an alternating current at the desired ultrasonic frequency, it produces a time varying mag- netic field which in turn induces eddy currents in the material under test, Fig. 4. If an external static magnetic field is present, the interaction of these eddy currents with the static magnetic field results in a magnetic volume force. This force results in the generation of a wave that propagates through the specimen. When the wave passes the region of the receiving EMAT, local eddy currents are induced in the conductive material, thus resulting in a time varying magnetic field which induces voltage in the coil. EMATs are one of the several types of noncontact ultrasonic transducers. EMATs have been successfully used for applications ranging from flaw detection to thickness gauging and stress measurement, [4].

\section{Numerical Evaluation and Experimental Measurement- EMAT Method}

The commercially available software OPERA-3D based on the Finite Element Method (FEM) is utilized for the purpose. Spatial configuration of the EMAT inspection is shown in Fig. 5. The problem deals with EMAT probe, placed above a material under investigation. The probe coil with dimensions is shown in Fig. 5. It is supplied with current density $J=1 \mathrm{~A} / \mathrm{mm}^{2}$ and frequency of the signal was $f=50 \mathrm{kHz}$. The second part of the EMAT probe is magnet with magnetic field which is orientedperpendicular to the surface. This configuration of the EMAT probe provides generation of a shear wave. The same configuration of the probe was used for experimental measurement,

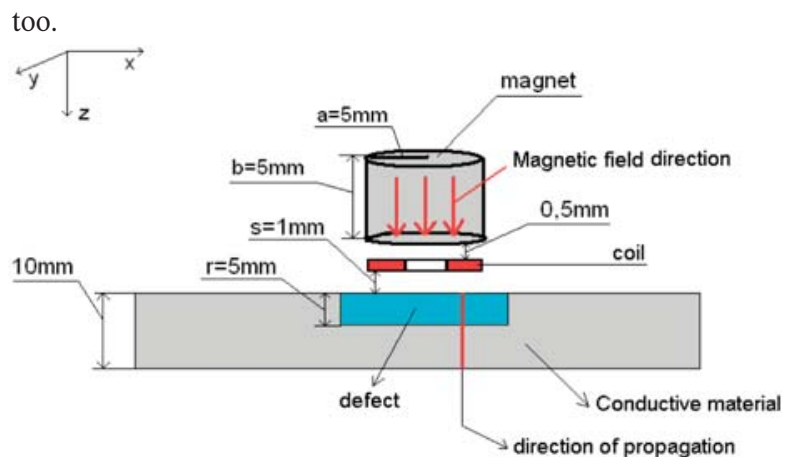

Fig. 5 Spatial configuration of the EMAT evaluation

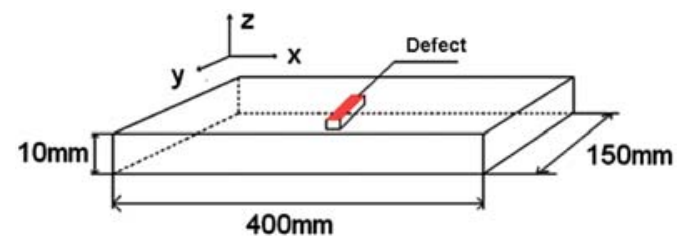

Fig. 6 Dimensions of the inspected material

Properties of the investigated material, dimensions and electromagnetic parameters of the material were set according to the real dimensions and electromagnetic parameters of the specimen, Fig. 6. The specimen was made of biomaterial SS 316 and has a conductivity of $\sigma=1.35 .106 \mathrm{~S} / \mathrm{m}$ and relative permeability of 


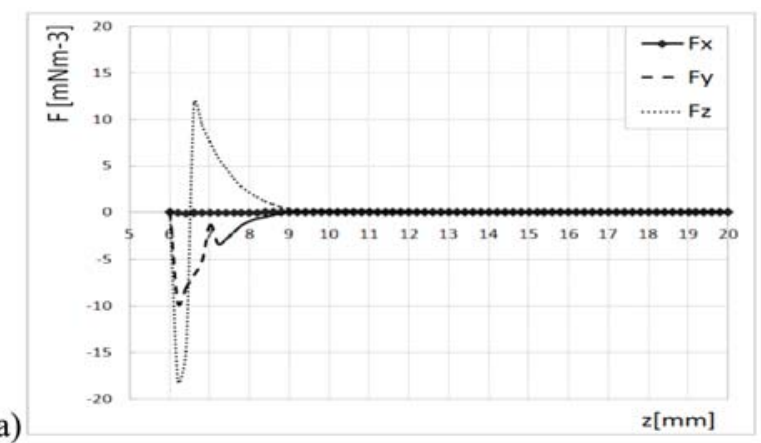

Fig. 7 Waveform of the Lorentz force components in direction to the material a) without defect, $b$ ) with defect $\mu \mathrm{r}=1$. The defect was localized in the middle of the samplel. The result of the numerical simulations shows the waveform of the Lorentz force for material with defect and without defect.

Based on the simulation results we can detect the defect in a material specimen. The $\mathrm{z}$ - component of the Lorentz force has the highest value and it represents shear waves, as it was mentioned in configuration EMAT probe.
The experimental measurements were realized using EMAT device and EMAT probe. During the inspection the probe was positioned on the specimen without defect and with defect. The defect in the specimen can be characterized as a volume defect. The reflected ultrasonic waves - echoes from the defect were detected.

Fig. 8a shows the transmitting signal which was sent into the inspected material without defect. The Fig. $8 \mathrm{~b}$ shows the echo re- a)

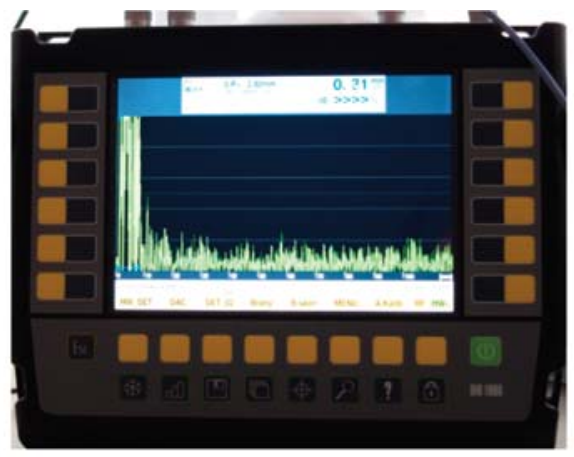

b)

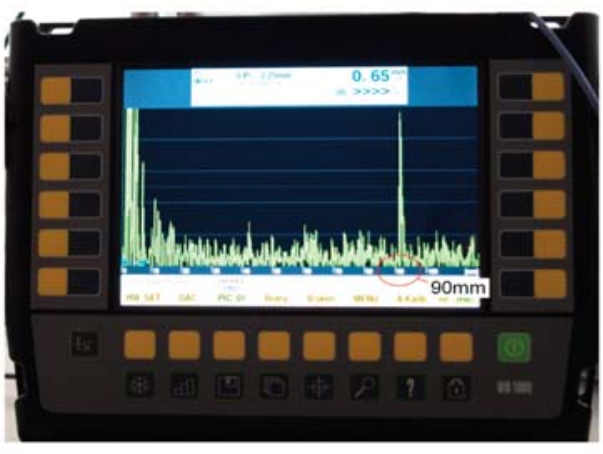

Fig.8 Signal detected from inspected material a) without defect, b) with defect

a)

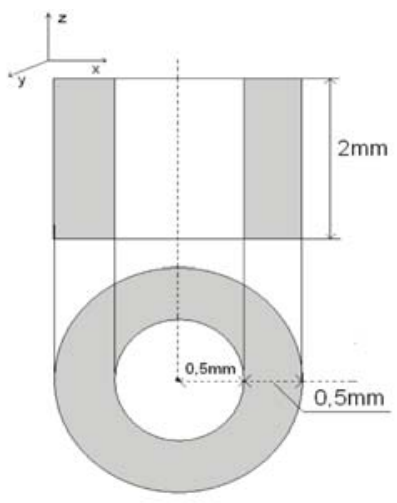

b)

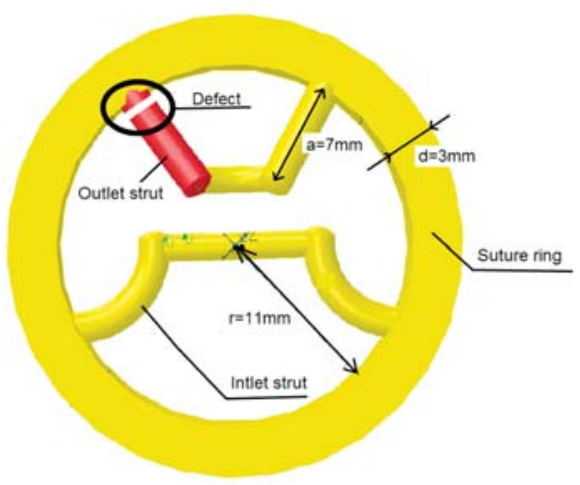

Fig. 9 a) Dimension of the coil, b) Dimension of the BSCC heart valve 
flected from the defect which was localized at the depth of $x=$ $=90 \mathrm{~mm}$ under the surface of the inspected material sample.

\section{Numerical Evaluation and Experimental Measurement - ECT Method}

Complicated design of the BSCC heart valve does not allow the scanning of the material the possibilities of sweep frequency eddy current inspection of BSCC are investigated in the paper using numerical means. Standard self-inductance pancake probe, shown in Fig. 9a, is used for the inspection. Dimensions of the probe are set up according to the dimensions of the heart valve. Dimensions of the catheter which would be used for encapsulations of the coil are considered as well. The probe is positioned $1 \mathrm{~mm}$ above the weld of the outer strut where a crack is considered to appear. The current density of the exciting signal is kept constant during the inspection at a value of $J=1 \mathrm{~A} / \mathrm{mm}^{2}$, while its frequency is changed in a wide range starting from $10 \mathrm{kHz}$ up to $500 \mathrm{kHz}$.

A complex model of the BSCC is developed. The dimensions and electromagnetic properties of the model are adjusted according to real dimensions and material properties of the BSCC heart valve. The dimensions of the heart valve are shown in Fig. 9b. The developed model considers the electromagnetic parameters of the $\mathrm{Co}-\mathrm{Cr}$ alloy (Hayness 25), the conductivity is adjusted to $\sigma=1.14 \cdot 10^{6}$ $\mathrm{S} / \mathrm{m}$ and the relative permeability to $\mu_{r}=1$.

The considered defect is localized at one end of the outlet strut, Fig. 9 b. The defect is non-conductive, i.e. fatigue crack, and it represents single leg fracture (SLF) of the BSCC. Dimensions, orientation and depth of the defect are adjusted according to the real conditions that may appear in the outlet strut of BSCC heart valve. The width of the modeled defect is set to $w=0.1 \mathrm{~mm}$ and its depth $d$ is varied from 0.1 up to $1.9 \mathrm{~mm}$ with a step of $0.2 \mathrm{~mm}$. The simulations are run for the intact outlet strut (IOS) as well as for SLF for comparison. Numerical simulations using the developed model are carried out to calculate the probe response signal.

The results of the numerical simulations of the model introduced above are presented in this section. Fig. 10 shows amplitude frequency spectrum of the probe response signal in absolute values for four cases. The curve denoted as IOS represents the sweep frequency response signal dependence for the intact outlet strut. The

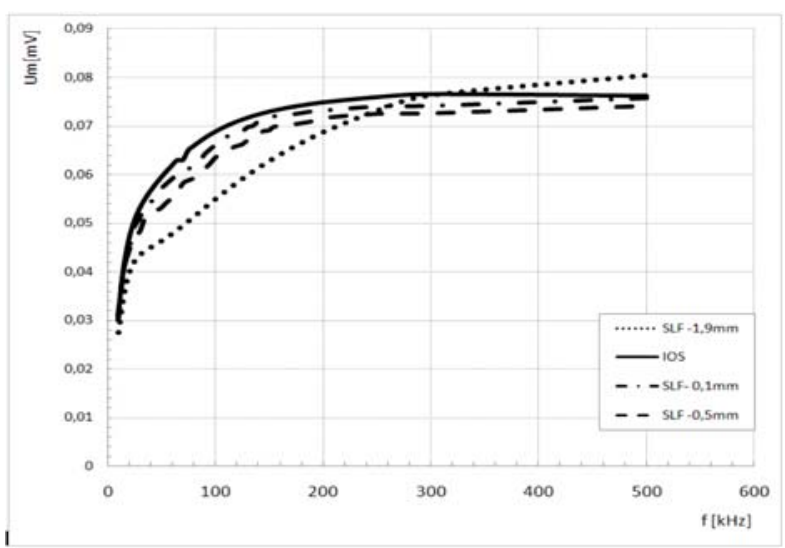

Fig. 10 Amplitude frequency spectrum of the probe response signal in absolute values

other three dependences are for the single leg fracture (SLF) with different depth of the defect, i.e. $0.1 \mathrm{~mm}, 0.5 \mathrm{~mm}$ and $1.9 \mathrm{~mm}$. The inductive component is dominant in the probe response signal and thus the phase frequency spectrum does not show almost any difference between the IOS and the SLF.

It is evident from the presented results that there is a clear difference in the amplitude frequency spectrum between the IOS and SLF mainly for the $1.9 \mathrm{~mm}$ which is significant within the frequency range $70 \mathrm{kHz}$ up to $150 \mathrm{kHz}$.

There was used the prototype of the BSCC heart valve in experimental part. The prototype was made from $\mathrm{CoCr}$ alloy type Hayness 25, Fig. 2. One prototype of the valve was without defect (intact outlet strut - IOS) and other prototype of the valve contained the defect on the one end of the outlet strut, Fig. 2. The depth of the defect was $d=0.6 \mathrm{~mm}$.

The eddy current testing probe with coil parameters $L=0.35$ $\mu \mathrm{H}$ and $N=600$ turns was used for the measurement The probe was positioned above the outlet strut with defect, in proximity of $s=1 \mathrm{~mm}$, Fig. 11a. The reference signal represents the signal from BSCC without defect.

Fig. 11b. shows a block diagram of the measurement, where are SG - signal generator, PA - power amplifier, $\mathrm{P}$ - probe, $R=0.5 \Omega$, HV - prototype of the heart valve, LIA - Lock- in amplifier with a)

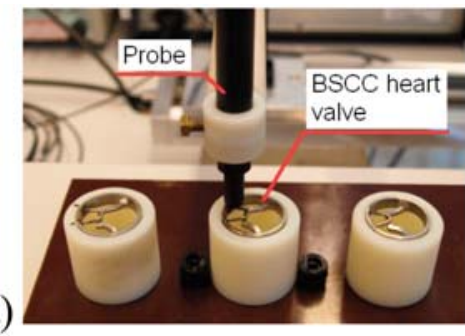

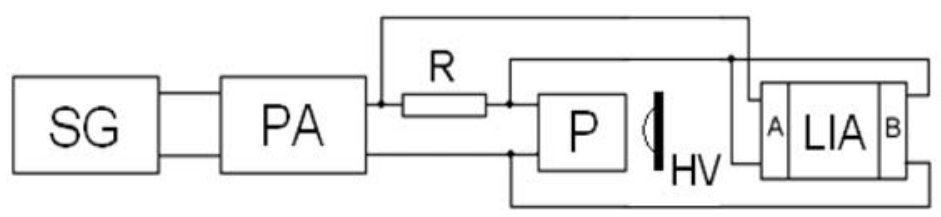

b)

Fig. 11: a) Orientation of the probe and prototype of the valve, b) The system block diagram of the measurement 
a)

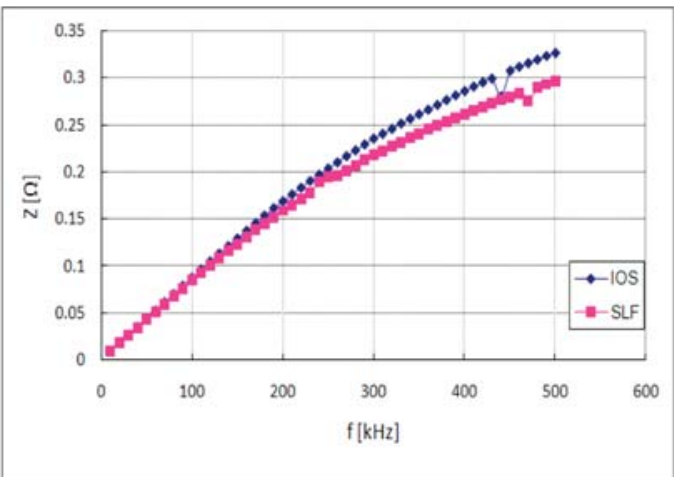

Fig. 12: a) Dependence of impedance amplitude on frequency, b) Dependence of impedance phase on frequency

b)

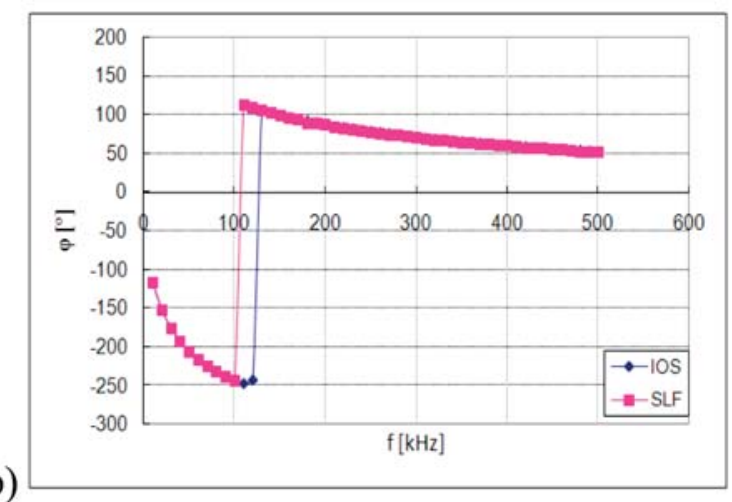

heart valve use. Both models of the biomaterial specimens and the artificial heart valve were developed and proposed methods were verified using numerical simulations and experimental measurements. The presented results of the numerical simulations and experiments clearly showed that the EMAT method is convenient for detection of volume cracks which could affect the biomaterials of prosthetic replacements. From the achieved results of the evaluation of BSCC heart valve using ECT method the differences in the amplitude frequency spectrum of the intact outlet strut and the strut mainly with deeper defect are evident.

\section{Acknowledgement}

This work was supported by the Slovak Research and Development Agency under the contract No. APVV-0194-07.

This work was supported by grant of the Slovak Grant Agency VEGA, project No. 1/0308/08.

The authors express their thanks to Prof. R. Grimberg for lending the valve prototypes.

The paper introduced new approaches for defect detection in a biomaterial for prosthetic replacement with focus on artificial

\section{References}

[1] VAN NEER, P.: The Bjork-Shiley valve: Detecting Broken Strut Using Standards Diagnostic Ultrasound Instruments, MSc Thesis,2005

[2] BORRERO, J. R., CURE, J., FABRE, N. J., ROSADO, E.: Mechanics of Prosthetic Heart Valves. Applications of Engineering

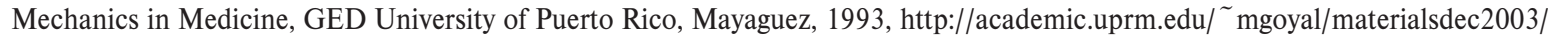
j03prostheticheartvalve.pdf

[3] JANOUSEK, L., MAREK, T., GOMBARSKA, D.: Eddy Current Non-destructive Testing of Conductive Materials, Communications - Scientific Letters of the University of Zilina, No. 8, 2006, pp. 29-33, ISSN 1335-4205

[4] MAXFIELD, B.: Electromagnetic Acoustic Transducer, Ultrasonic Nondestructive Evaluation, Engineering and Biological Material Characterization, Chapter 8, CRC Press LLC 2004. 